\title{
Kommentar zum Kommentar von F. Steger zum Beitrag „Arbeits(zeit)schutz nach Art der BRD - am Beispiel der Covid-19-ArbZV“
}

\section{Friedhelm Nachreiner $^{1}$}

Angenommen: 11. Juni 2021 / Online publiziert: 4. Juli 2021

(c) Springer-Verlag GmbH Deutschland, ein Teil von Springer Nature 2021

Man kann Steger nur Recht geben, wenn er feststellt, dass eigentlich keiner ernsthafte Zweifel an der Bedeutung des Arbeitsschutzes anmelden dürfte. Dummerweise sind aber verbale Bekundungen und faktisches Handeln nicht immer konsistent, wie die beiden als Beispiele angeführten Verordnungen des BMAS zeigen. Es ging mir bei meinem Beitrag nicht primär um das COVID-19 Problem, das diente nur als Demonstrationsbeispiel für die Verletzung von Grundlagen des Arbeitsschutzes durch den Verordnungsgeber, mit der Möglichkeit des Versuchs der Legitimierung der Verletzungen durch hoch- oder höherrangige Ziele. Dieselbe Missachtung arbeitswissenschaftlicher Erkenntnisse findet sich ja auch in anderen Zusammenhängen, wie ich am Beispiel der Offshore-ArbZV aus 2013 gezeigt habe und für die es keine Zeitzwänge gab, die es aber den Arbeitnehmervertretern schwer macht, gesetzliche Vorgaben umzusetzen, wie etwa die der im ArbZG § 6 (1) geforderten Berücksichtigung der gesicherten arbeitswissenschaftlichen Erkenntnisse. Im Gegensatz zur COVID-19-ArbZV ging es bei dieser Verordnung primär um wirtschaftliche Interessen und Interessen der Verwaltungsvereinfachung und der Entlastung der Verwaltung von der Verantwortung für die Verletzung arbeitswissenschaftlicher Erkenntnisse im Rahmen der Genehmigung traditioneller Arbeitszeitgestaltungsmuster.

Beide Beispiele belegen auf der Basis evidenzbasierter empirischer und theoretischer Zusammenhänge sehr deutlich die mit der Missachtung dieser Befunde verbundenen Risiken. Man mag das narrativ, nachvollziehbar oder plausibel finden, ich halte die vorliegende Evidenzbasis für die hier verfolgten Ziele einer angemessenen Berücksichtigung arbeitswissenschaftlicher Erkenntnisse im Arbeitsschutz für

Friedhelm Nachreiner

friedhelm.nachreiner@gawo-ev.de

1 Gesellschaft für Arbeits-, Wirtschafts- und Organisationspsychologische Forschung e. V., Achterdiek 50, 26131 Oldenburg, Deutschland hinreichend und bin gerne bereit, dies bei Bedarf detailliert zu belegen.

Übrigens modellieren wir nicht nur wegen angeblich oder für bestimmte Fragestellungen z.T. tatsächlich fehlender Daten. Ich habe von meinem akademischen Lehrer, Mediziner und Psychologe, gelernt, dass man Beschäftigte nicht ins Verderben rennen lassen darf, wenn man aversive Konsequenzen theoretisch und auf der Basis des vorhandenen Wissens erwarten muss, nur um eine Hypothese zu prüfen oder zu bestätigen. Glücklicherweise gibt es dazu heute in einigen Bereichen statistisch gut basierte und abgesicherte Prognose- und Modellierungstechniken, die z.T. frei im Netz verfügbar sind - auch für diejenigen, die normative Regelungen produzieren.

Ich kann dem Kommentator allerdings nur zustimmen, wenn er fordert, die vorhandene arbeitswissenschaftliche Expertise unbedingt in die politischen Entscheidungsprozesse einzubinden - und, wie ich ergänzend hinzufügen möchte, nicht nur auf betriebswirtschaftliche oder organisatorische Argumente einzugehen. Offensichtlich berücksichtigen diese nicht die Komplexität und Nichtlinearitäten der gegebenen Zusammenhänge im Arbeitsverhalten, wie sich beispielsweise auch in Berichten der „Wirtschaftsweisen“ regelmäßig zeigen lässt, hier etwa am Beispiel der Flexibilität von Arbeitszeiten.

Für meinen Beitrag will ich gerne noch darauf hinweisen, dass es m.E. nicht die enge zeitliche Bindung (wie wir als Arbeitswissenschaftler die Belastungsbedingung kennzeichnen würden) war, die ausschlaggebend für die nach den vorliegenden arbeitswissenschaftlichen Erkenntnissen falschen Entscheidungen bei der COVID-19-ArbZV waren, sondern eher (sozial)politische Prioritäten. Dass man aus einer personellen Unterdeckung auch durch intensivere Nutzung der vorhandenen personellen Ressourcen keine personelle Überdeckung erreichen kann, ist hinlänglich, auch aus der Geschichte, bekannt. Auch darauf habe ich hingewiesen. Und auch moderne Rechnersysteme sind bisher nicht in der Lage, durch die Arbeitszeitgestaltung und 
deren (einseitige) Optimierung aus einer personellen Unterdeckung eine Überdeckung zu generieren, auch wenn der Wunsch danach in Beratungsprojekten immer wieder auftaucht.

Dass es eher am Wandel von Werten oder Prioritäten liegt, die den Arbeitsschutz hinten runter fallen lassen, zeigt sich auch in einigen jüngeren Publikationen, wenn es etwa, wie bei der BDA (2021) in deren Positionspapier unter dem Thema „Die Arbeitszeit der Zukunft braucht mehr Spielräume“ als aus deren Sicht dringende Forschungsfrage heißt, „Wie lassen sich die arbeitswissenschaftlichen Kriterien zur Arbeitszeit- und Schichtplangestaltung mit den veränderten Anforderungen der Arbeitswelt 4.0 (besser) in Einklang bringen? Wie müssen diese arbeitswissenschaftlichen Kriterien angepasst werden?" (Hervorhebung vom Autor) Genau da scheint mir das Problem zu liegen: es geht weniger um die Anpassung der Arbeit an den Mensch und seine „Funktionsweise“ als um die Anpassung des Menschen an wirtschaftliche und technologische Anforderungen. Mit Ergonomie (und vielleicht auch mit Ethik?) hat das m. E. nicht allzu viel zu tun, wie die Beispiele in meinem Beitrag belegen. Vielleicht sollten wir uns daher doch noch einmal ein paar intensivere Gedanken über die Bedeutung und Facetten des Arbeitsschutzes in der BRD machen und darüber diskutieren. Wenn mein Beitrag dazu anregen kann, und das glaube ich in dem Kommentar dankenswerter Weise ebenfalls erkennen zu können, hätte er sein Ziel, zumindest teilweise, erreicht.

\section{Literatur}

Bundesvereinigung der Deutschen Arbeitgeberverbände (BDA) (2021) Arbeitsforschung 2021+: Welche Forschungsfragen bewegen die Arbeitgeber und wie sieht die Arbeitswelt der Zukunft aus? Positionspapier. Z Arb Wiss 75:127-136. https://doi.org/10.1007/ s41449-021-00240-3 